\title{
Nonequilibrium Kubo Formula of Finite Conductor Connected to Reservoirs based on Keldysh Formalism
}

\author{
Tatsuya Fujii \\ Institute for Solid State Physics, University of Tokyo, Kashiwa 277-8581, Japan
}

(Dated: July 8, 2021)

\begin{abstract}
We show that the density matrix for a finite conductor attached to reservoirs obtained by Keldysh formalism is of MacLennan-Zubarev form. On the basis of the fact that the density matrix is the invariant part proposed by Zubarev, it is shown that Keldysh formalism may describe the irreversible processes and steady-state feature of the system. An important consequence of the MacLennanZubarev form of the density matrix is a generalization of the Kubo formula in a nonequilibrium case. On the basis of the result, we propose the formula of shot noise and a nonequilibrium identity between differential conductance, noise power and shot noise as a generalized Nyquist-Johnson relation.
\end{abstract}

PACS numbers: $73.63 . \mathrm{Kv}$

\section{INTRODUCTION}

Recently many-body problems in nonequilibrium steady states have been studied in mesoscopic devices. Keldysh formalism $1,2,3,4,5$ has been applied to study various systems. It has been used successfully to calculate observable quantities such as differential conductance and noise power.

However, in mesoscopic systems with electron correlation, progress has been limited even in the basic understanding of nonequilibrium steady states. Of course the linear response theory is well established. In mesoscopic systems, the Kubo formula leads to the NyquistJohnson relation between conductance and noise power. However, there has been little discussion on the generalization of the Kubo formula and the relation between physical quantities in many-body systems in a nonequilibrium situation.

One possible means of discussing general features under the nonequilibrium condition independent of different systems may be to consider density matrices. In this study, we consider a conductor in series with equilibrium reservoirs with a finite bias voltage. There is an approach to formulating the density matrix of this system ${ }^{6.7}$, in which the density matrix is given by Keldysh formalism, although the term Keldysh formalism is not used. The obtained density matrix is similar to the equilibrium ensemble. The density matrix is expressed as an infinite series of operators, which are generated by the perturbative expansion of the s-matrix. Therefore, the density matrix has remained to be a formal expression. The generalization of the Kubo formula under a nonequilibrium condition has not been successful.

On the other hand, there has been progress by a different method. By using a rigorous approach of the $\mathrm{C}^{*}$ algebra, a density matrix of MacLennan-Zubarev form has been obtained for a finite system attached to reservoirs 8.9 . Concerning the transport properties of a one-dimensional noninteracting lattice or a quantum dot using the slave-boson technique, it has been reported that a current-current correlation function is given by the sum of differential conductance and an additional term. However, the physical picture and origin of the additional term have not been clarified ${ }^{8,10}$.

In this study we show that the Keldysh formalism of the density matrix of a finite conductor attached to reservoirs is of MacLennan-Zubarev form. We show that Keldysh formalism has sufficient features to describe the nonequilibrium steady state. We finally find that the MacLennan-Zubarev form of the density matrix leads to a nonequilibrium Kubo formula. On the basis of this form, we propose a nonequilibrium identity between differential conductance, noise power and shot noise, which is an extended Nyquist-Johnson relation in correlated systems in a nonequilibrium case.

\section{SYSTEM AND HAMILTONIAN}

We discuss a finite conductor attached to the left and right infinite reservoirs through boundary couplings. The system Hamiltonian is defined as

$$
H \equiv H_{c}+H_{L}+H_{R}+H_{c L, R},
$$

where $H_{c}, H_{L, R}$ and $H_{c L, R}$ describe the conductor, left and right reservoirs, and boundary couplings, respectively. The conductor part of the Hamiltonian is given by the sum of the noninteracting and interacting parts, $H_{c} \equiv H_{c 0}+H_{c 1}$. We stress that, in the following analysis, the correlation effect is included. Generally, in the preparation of the Keldysh Green function, the system Hamiltonian $H$ is divided into the nonperturbative and perturbative terms $11,12,13$ as

$$
H=H_{0}+H_{1}
$$

The nonperturbative term $H_{0} \equiv H_{c 0}+H_{L}+H_{R}$ describes three independent systems, which are the noninteracting conductor and infinite reservoirs. The perturbative term $H_{1} \equiv H_{c L, R}+H_{c 1}$ is the sum of boundary couplings and 
the interaction inside the conductor. In many cases, $H_{c 1}$ is treated using a perturbation theory to calculate the Green function in Keldysh formalism. Notice that the following results do not depend on the method used to divide $H$ into $H_{0}$ and $H_{1}$, which will be shown explicitly in section 4.

Clearly, the energy scale of $H_{1}$ is much smaller than that of $H_{0}$ because $H_{1}$ describes the effects in the finite conductor. Thus, it may be expected that the expansion of the s-matrix is a well-behaved series.

\section{ADIABATIC SWITCHING-ON OF $H_{1}$ IN KELDYSH FORMALISM}

Let us begin with the Keldysh formalism of a finite conductor connected to infinite reservoirs ${ }^{11,12,13}$. Generally, the discussion based on Keldysh formalism is concentrated on the Keldysh contour of the s-matrix and the Keldysh Green function. We carefully analyze the time dependence of Keldysh formalism in which the perturbative term is imposed adiabatically.

In the initial state at $t_{0}=-\infty$, the conductor and infinite reservoirs are decoupled in equilibrium state, where the initial density matrix is supposed to be given by $\rho_{0} \equiv e^{-\beta\left(H_{0}-\mu N_{c}-\mu_{L} N_{L}-\mu_{R} N_{R}-\Omega_{0}\right)}$ and $\Omega_{0}$ is the initial thermodynamic potential. The reservoirs are tuned to different chemical potentials. For simplicity, we define $\left(\mu_{L}+\mu_{R}\right) / 2 \equiv \mu \equiv 0$ and $\left(\mu_{L}-\mu_{R}\right) / 2 \equiv e V / 2$. We can rewrite the initial density matrix as

$$
\rho_{0}=e^{-\beta\left(H_{0}-e V / 2\left(N_{L}-N_{R}\right)-\Omega_{0}\right)} .
$$

In the following, we assume $\left[H_{0}, N_{L}-N_{R}\right]=0$.

The perturbative term is turned on adiabatically as $g e^{-\epsilon|t|} H_{1}$, where $g$ is introduced to tune the magnitude of the coupling constant, and $\epsilon$ is a positive infinitesimal number. We define the total Hamiltonian as

$$
H_{\epsilon} \equiv H_{0}+g e^{-\epsilon|t|} H_{1} .
$$

The time evolution of the system obeys the Neumann equation

$$
\mathrm{i} \frac{\partial}{\partial t} \rho_{\epsilon}(t)=\left[H_{\epsilon}, \rho_{\epsilon}(t)\right],
$$

where the initial state is given by eq. (3) , and $[A, B] \equiv$ $A B-B A$ is the commutation relation. The formal solution of the Neumann equation is given by

$$
\begin{aligned}
\rho_{\epsilon}(t) & =U_{\epsilon}\left(t, t_{0}\right) \rho_{\epsilon}\left(t_{0}\right) U_{\epsilon}\left(t_{0}, t\right) \\
& =e^{-\mathrm{i} H_{0} t} S_{\epsilon}\left(t, t_{0}\right) e^{\mathrm{i} H_{0} t_{0}} \rho_{\epsilon}\left(t_{0}\right) e^{-\mathrm{i} H_{0} t_{0}} S_{\epsilon}\left(t_{0}, t\right) e^{\mathrm{i} H_{0} t} .
\end{aligned}
$$

$U_{\epsilon}\left(t, t_{0}\right)$ or its equivalent $S_{\epsilon}\left(t, t_{0}\right)$ satisfies

$$
\begin{aligned}
& \mathrm{i} \frac{\partial}{\partial t} U_{\epsilon}\left(t, t_{0}\right)=H_{\epsilon} U_{\epsilon}\left(t, t_{0}\right) \\
& \mathrm{i} \frac{\partial}{\partial t} S_{\epsilon}\left(t, t_{0}\right)=g e^{-\epsilon|t|} H_{1}(t) S_{\epsilon}\left(t, t_{0}\right)
\end{aligned}
$$

where $U_{\epsilon}\left(t, t_{0}\right)=e^{-\mathrm{i} H_{0} t} S_{\epsilon}\left(t, t_{0}\right) e^{\mathrm{i} H_{0} t_{0}}$. In the following analysis, we employ the explicit forms $U_{\epsilon}(t, 0)$ and $S_{\epsilon}(t, 0)$. Although the expressions of $U_{\epsilon}\left(t, t_{0}\right)$ and $S_{\epsilon}\left(t, t_{0}\right)$ are well known, here we show them explicitly for later use as

$$
\begin{aligned}
U_{\epsilon}\left(t, t_{0}\right)=1+\sum_{n=1}^{\infty} \frac{(-\mathrm{i})^{n}}{n !} \int_{t_{0}}^{t} \mathrm{~d} t_{1} \cdots \mathrm{d} t_{n} T\left[H_{\epsilon 1} \cdots H_{\epsilon n}\right], \\
S_{\epsilon}\left(t, t_{0}\right)=1+\sum_{n=1}^{\infty} \frac{(-\mathrm{i} g)^{n}}{n !} \int_{t_{0}}^{t} \mathrm{~d} t_{1} \cdots \mathrm{d} t_{n} \\
e^{-\epsilon\left(\left|t_{1}\right|+\cdots\left|t_{n}\right|\right)} T\left[H_{1}\left(t_{1}\right) \cdots H_{1}\left(t_{n}\right)\right],
\end{aligned}
$$

where $T$ is the time-ordering operator and $H_{\epsilon n} \equiv H_{0}+$ $g e^{-\epsilon\left|t_{n}\right|} H_{1}$.

The expectation value of any operator $\mathcal{O}$ is given by

$$
\operatorname{Tr}\left\{\mathcal{O} \rho_{\epsilon}(t)\right\}=\operatorname{Tr}\left\{\mathcal{O}_{H}(t) \bar{\rho}_{\epsilon}\right\} \equiv\left\langle\mathcal{O}_{H}(t)\right\rangle,
$$

where $\bar{\rho}_{\epsilon} \equiv \rho_{\epsilon}(0)$. The Heisenberg representation is defined by

$$
\mathcal{O}_{H}(t)=U_{\epsilon}(0, t) \mathcal{O} U_{\epsilon}(t, 0)=S_{\epsilon}(0, t) \mathcal{O}(t) S_{\epsilon}(t, 0),
$$

where $\mathcal{O}(t)=e^{\mathrm{i} H_{0} t} \mathcal{O} e^{-\mathrm{i} H_{0} t}$ is the interaction representation. The physical quantities are defined in the limit $g \rightarrow 1, \epsilon \rightarrow 0$. On the basis of the observation that $\lim _{g \rightarrow 1, \epsilon \rightarrow 0} H_{\epsilon}=H$. In Keldysh formalism, $\mathcal{O}$ is supposed to exist in the limit $g \rightarrow 1, \epsilon \rightarrow 0$.

\section{MACLENNAN-ZUBAREV FORM}

Let us focus on the density matrix $\bar{\rho}_{\epsilon}$ defined by $\bar{\rho}_{\epsilon}=$ $S_{\epsilon}(0,-\infty) \rho_{0} S_{\epsilon}(-\infty, 0)$, which may be rewritten as

$$
\begin{aligned}
\bar{\rho}_{\epsilon}=\exp \left\{-\beta S_{\epsilon}(0,-\infty)\left(H_{0}\right.\right. & -e V / 2\left(N_{L}-N_{R}\right) \\
& \left.\left.-\Omega_{0}\right) S_{\epsilon}(-\infty, 0)\right\} .
\end{aligned}
$$

This is simply a formal expression. In standard calculations of Keldysh formalism, the s-matrix is almost always perturbatively expanded. In practice, we can sometimes estimate observable quantities based on some approximations even if we do not have the complete knowledge of the density matrix. In this paper, we try to analyze the structure of the density matrix, which leads to a deeper understanding of the nonequilibrium properties of mesoscopic systems.

Here, in order to study $\bar{\rho}_{\epsilon}$, we derive a key relation for

$$
\bar{A}_{\epsilon} \equiv S_{\epsilon}(0,-\infty) A S_{\epsilon}(-\infty, 0),
$$

where the operator $A$ satisfies $\left[A, H_{0}\right]=0$. We notice concerning $A=H_{0}$ that a useful expression of $\bar{A}_{\epsilon}$ is

$$
\begin{aligned}
& S_{\epsilon}(0,-\infty) H_{0} S_{\epsilon}(-\infty, 0) \\
& \quad=H_{0}+g H_{1}-\mathrm{i} \epsilon g \frac{\partial S_{\epsilon}(0,-\infty)}{\partial g} S_{\epsilon}(-\infty, 0),
\end{aligned}
$$


which is obtained using the Gell-Mann and Low theorem 14,15 . In the following, we derive a different expression for the arbitrary operator $A$ that satisfies
$\left[A, H_{0}\right]=0$.

First, we discuss the commutation relation between $A$ and the s-matrix.

$$
\begin{gathered}
{\left[A, S_{\epsilon}(0,-\infty)\right]=\sum_{n=1}^{\infty} \frac{(-\mathrm{i} g)^{n}}{n !} \int_{-\infty}^{0} \mathrm{~d} t_{1} \cdots \mathrm{d} t_{n} e^{-\epsilon\left(\left|t_{1}\right|+\cdots+\left|t_{n}\right|\right)}(\mathrm{i} g)^{-1} n \sum_{l=1}^{n}{ }_{n-1} C_{l-1}} \\
T\left[H_{1}\left(t_{1}\right) \cdots H_{1}\left(t_{l-1}\right)\right] J_{A}\left(t_{l}\right) T\left[H_{1}\left(t_{l+1}\right) \cdots H_{1}\left(t_{n}\right)\right],\left\{t_{1}, \cdots, t_{l-1}\right\}>t_{l}>\left\{t_{l+1}, \cdots, t_{n}\right\} .
\end{gathered}
$$

The "current" corresponding to the operator $A$ is defined as

$$
J_{A H}(t) \equiv-\frac{\partial}{\partial t} A_{H}(t) .
$$

The calculation of eq.15) can be performed as follows: We expand the s-matrix and consider the $n$th term in the series. Among the $n$ ! possible time orderings, a certain term of $t_{p}>t_{q}>\cdots>t_{r}$ is chosen. The commutation relation $\left[A, H_{1}\left(t_{p}\right) H_{1}\left(t_{q}\right) \cdots H_{1}\left(t_{r}\right)\right]$ may be rewritten using $\left[A, H_{1}(t)\right]=(\mathrm{i} g)^{-1} J_{A}(t)$ for $\left[A, H_{0}\right]=0$. With reference to the time variable $t_{l}$ of $J_{A}$, we can categorize times larger $\left\{t_{1}, \cdots, t_{l-1}\right\}$ and smaller $\left\{t_{l+1}, \cdots, t_{n}\right\}$ than $t_{l}$. We sum possible time orderings except $J_{A}\left(t_{l}\right)$ while taking advantage of dummy integration variables. Finally, we obtain

$$
\left[A, S_{\epsilon}(0,-\infty)\right]=-\int_{-\infty}^{0} \mathrm{~d} t e^{-\epsilon|t|} J_{A H}(t) S_{\epsilon}(0,-\infty)
$$

It is easy to rewrite this as

$$
\begin{aligned}
\bar{A}_{\epsilon} & =S_{\epsilon}(0,-\infty) A S_{\epsilon}(-\infty, 0) \\
& =A+\int_{-\infty}^{0} \mathrm{~d} t e^{-\epsilon|t|} J_{A H}(t) .
\end{aligned}
$$

Integration by parts in eq.(18) also gives,

$$
\bar{A}_{\epsilon}=\epsilon \int_{-\infty}^{0} \mathrm{~d} t e^{-\epsilon|t|} A_{H}(t) .
$$

Note that $\bar{A}_{\epsilon}$ is the invariant part of the operator $A$ defined by Zubarev $\stackrel{16}{\underline{16}}$. The concept of the invariant part will play an essential role in the next section.

By using eq. (18), the density matrix of eq. (12) can be evaluated as

$$
\begin{aligned}
\bar{\rho}_{\epsilon} & =\exp \left\{-\beta\left(H_{0}+\int_{-\infty}^{0} \mathrm{~d} t e^{-\epsilon|t|} J_{e H}(t)\right.\right. \\
& \left.\left.-e V / 2\left(N_{L}-N_{R}\right)-V \int_{-\infty}^{0} \mathrm{~d} t e^{-\epsilon|t|} J_{c H}(t)-\Omega_{0}\right)\right\},
\end{aligned}
$$

where $J_{e H}(t)$ and $J_{c H}(t)$ are the energy change and charge current, respectively, which are obtained by setting $A=H_{0}$ and $A=e / 2\left(N_{L}-N_{R}\right)$ in eq. (16).

The obtained density matrix is nothing but a type of MacLennan-Zubarev form of the density matrix 16.17, which is characterized by the time-integral of the "current" on the negative semi-infinite interval. We have obtained this form for the density matrix by dividing the system into $H_{0}=H_{c 0}+H_{L}+H_{R}$ and $H_{1}=H_{c L, R}+H_{c 1}$. On the other hand, for the case of $H_{0}=H_{L}+H_{R}$ and $H_{1}=H_{c L, R}+H_{c 1}+H_{c 0}$, it was obtained using a rigorous approach of a $\mathrm{C}^{*}$ algebra ${ }^{9}$. Here, let us show that the obtained density matrix does not depend on the method used to divide the Hamiltonian.

In eq. (18),

$$
S_{\epsilon}(0,-\infty) H_{0} S_{\epsilon}(-\infty, 0)=H_{0}+\int_{-\infty}^{0} \mathrm{~d} t e^{-\epsilon|t|} J_{e H}(t)(21)
$$

seems to be dependent on the method used to divide $H$ into $H_{0}$ and $H_{1}$.

As pointed out previously, $S_{\epsilon}(0,-\infty) H_{0} S_{\epsilon}(-\infty, 0)$ is rewriten as eq.(14). On the right-hand side of eq. (14), $H_{0}+g H_{1}$ is clearly independent of the method used to divide $H$ into $H_{0}$ and $H_{1}$ in the limit $g \rightarrow 1, \epsilon \rightarrow 0$; hence, we discuss the last term

$$
\Delta \Omega_{\epsilon} \equiv \mathrm{i} \epsilon g \frac{\partial S_{\epsilon}(0,-\infty)}{\partial g} S_{\epsilon}(-\infty, 0) .
$$

Using $S_{\epsilon}(0,-\infty)=U_{\epsilon}(0,-\infty)$ and $U_{\epsilon}(0,-\infty)=$ $U_{\epsilon}(-\infty, 0)$, we obtain

$$
\Delta \Omega_{\epsilon}=\epsilon \sum_{n=1}^{\infty} \frac{(-\mathrm{i} g)^{n-1}}{n !} \int_{-\infty}^{\infty} \mathrm{d} t_{1} \cdots \mathrm{d} t_{n} T\left[H_{\epsilon 1} \cdots H_{\epsilon n}\right]
$$

Now, we assume that $H$ is given by the sum of the operators $A, B$ and $C$ as $H=A+B+C$. We compare two cases: $H_{0}=A+B, H_{1}=C$ and $H_{0}^{\prime}=A, H_{1}^{\prime}=B+C$. From $H_{\epsilon n}=H_{0}+g e^{-\epsilon\left|t_{n}\right|} H_{1}$, we obtain

$$
H_{\epsilon n}=H_{\epsilon n}^{\prime}+\left(1-g e^{-\epsilon\left|t_{n}\right|}\right) B .
$$

The last term $\left(1-g e^{-\epsilon\left|t_{n}\right|}\right) B$ which is dependent on the method used to divide the Hamiltonian vanishes in the 
limit $g \rightarrow 1, \epsilon \rightarrow 0$, hence $\Omega_{\epsilon}$ remains the same. Thus, the density matrix in eq.(20) does not depend on a division of the Hamiltonian in the limit $g \rightarrow 1, \epsilon \rightarrow 0$. Therefore, we can conclude that the form obtained using the $\mathrm{C}^{*}$ algebra is identical with the present form obtained using Keldysh formalism.

\section{CHARACTERISTIC FEATURE OF NONEQUILIBRIUM STEADY STATE}

The fact that $\bar{\rho}_{\epsilon}$ is the invariant part of the density matrix leads to the understanding of the dissipation in the system and also of the mechanism needed to reach a steady state. These are the points clarified by Zubarev ${ }^{16}$. However, in view of its importance, we briefly repeat the essential parts of the arguments explicitly using Keldysh formalism.

\subsection{Invariant part of density matrix and causality condition}

The Neumann equation in eq.(5) is rewritten as

$$
\mathrm{i} \frac{\partial}{\partial t} \ln \rho_{\epsilon}(t)=\left[H_{\epsilon}, \ln \rho_{\epsilon}(t)\right]
$$

By using eq.(18), $\ln \bar{\rho}_{\epsilon}$ can be also written as

$$
\ln \bar{\rho}_{\epsilon}=\ln \rho_{0}+\int_{-\infty}^{0} \mathrm{~d} t e^{-|\epsilon| t} J_{\ln \rho_{0 H}}(t) .
$$

where $J_{\ln \rho_{0 H}}(t)$ is defined using eq. (16) for $A=\ln \rho_{0}$. Corresponding to eq.(19), we rewrite it as

$$
\begin{aligned}
\lim _{\epsilon \rightarrow 0} \ln \bar{\rho}_{\epsilon} & =\lim _{\epsilon \rightarrow 0} \epsilon \int_{-\infty}^{0} \mathrm{~d} t e^{-|\epsilon| t} \ln \rho_{0 H}(t) \\
& =\lim _{T_{a} \rightarrow \infty} \frac{1}{T_{a}} \int_{-T_{a}}^{0} \mathrm{~d} t \ln \rho_{0 H}(t) .
\end{aligned}
$$

We find that $\ln \bar{\rho}_{\epsilon}$ is given by the long-time average of the logarithm of evolution of the initial density matrix.

This is nothing but Zubarev's definition of a nonequilibrium statistical operator ${ }^{16}$. He has pointed out that the entropy production is zero in the local equilibrium state; hence, the local equilibrium ensembles cannot describe irreversible processes. To incorporate dissipation, the idea of coarse graining has been introduced. He has defined the nonequilibrium statistical operator as the long-time average of entropy which is logarithm of the local equilibrium ensemble. The time average has been defined at the negative semi-infinite interval, namely, the causality condition has been imposed. Thus, the entropy production has been proved to be positive, although the proof itself was limited in the linear response regime.

Therefore, the adiabatic turning on of $H_{1}$ precisely corresponds to taking the invariant part with the causality condition. Therefore, we conclude that the adiabatic description of Keldysh formalism may describe irreversible processes.

\subsection{Steady-state feature of time-correlation functions}

Based on the fact that $\bar{\rho}_{\epsilon}$ is the invariant part, we will show that the steady state is described by the adiabatic switching on of Keldysh formalism in the limit $\epsilon \rightarrow 0$.

We start our discussion with charge current. Following similar calculations to obtain eq.(17), we show that the commutation relation between $\bar{\rho}_{\epsilon}$ and $U_{\epsilon}(0, t)$ is expressed as

$$
\left[\bar{\rho}_{\epsilon}, U_{\epsilon}(0, t)\right]=-\int_{t}^{0} \mathrm{~d} t_{1} J_{\bar{\rho}_{\epsilon} H}\left(t_{1}\right) U_{\epsilon}(0, t),
$$

where $J_{\bar{\rho}_{\epsilon} H}\left(t_{1}\right)$ is defined by

$$
\begin{aligned}
J_{\bar{\rho}_{\epsilon} H}\left(t_{1}\right) & \equiv-\frac{\partial}{\partial t_{1}} \bar{\rho}_{\epsilon H}\left(t_{1}\right) \\
& =U_{\epsilon}\left(0, t_{1}\right) \mathrm{i}\left[\bar{\rho}_{\epsilon}, H_{\epsilon 1}\right] U_{\epsilon}\left(t_{1}, 0\right) .
\end{aligned}
$$

From eq.(28), we rewrite the expectation value of the current as

$$
\begin{aligned}
& \operatorname{Tr}\left\{\bar{\rho}_{\epsilon} J_{c H}(t)\right\}=\operatorname{Tr}\left\{U_{\epsilon}(t, 0) \bar{\rho}_{\epsilon} U_{\epsilon}(0, t) J_{c}\right\} \\
& =\operatorname{Tr}\left\{\bar{\rho}_{\epsilon} J_{c}\right\}+\int_{0}^{t} \mathrm{~d} t_{1} \operatorname{Tr}\left\{J_{\bar{\rho}_{\epsilon} H}\left(t_{1}\right) J_{c H}(t)\right\} .
\end{aligned}
$$

We find that $J_{\bar{\rho}_{\epsilon} H}\left(t_{1}\right)$ is a key quantity for determining the time dependence of charge current. $J_{\bar{\rho}_{\epsilon} H}\left(t_{1}\right)$ is time derivative of the Heisenberg representation of the invariant part $\bar{\rho}_{\epsilon}$, which is one of the local integrals of motion defined by Zubarev $\stackrel{16}{ }$.

Here, we discuss $\left[\bar{\rho}_{\epsilon}, H_{\epsilon 1}\right]$ in eq.(29). By using the invariant part of $\bar{\rho}_{\epsilon}$ in eq.(19), the commutation relation is rewritten as

$$
\begin{aligned}
{\left[\bar{\rho}_{\epsilon}, H_{\epsilon 1}\right]=-\mathrm{i} \epsilon } & \int_{-\infty}^{0} \mathrm{~d} t_{2} e^{-\epsilon\left|t_{2}\right|} J_{\rho_{0} H}\left(t_{2}\right) \\
& +\epsilon \int_{-\infty}^{0} \mathrm{~d} t_{2} g_{\epsilon}\left[\rho_{0 H}\left(t_{2}\right), H_{\epsilon 1}\right],
\end{aligned}
$$

where we used

$$
\begin{aligned}
& {\left[\rho_{0 H}\left(t_{2}\right), H_{\epsilon 1}\right]} \\
& \quad=\left[\rho_{0 H}\left(t_{2}\right), H_{\epsilon 2}\right]+\left[\rho_{0 H}\left(t_{2}\right), H_{\epsilon 1}-H_{\epsilon 2}\right] \\
& \quad=-\mathrm{i} J_{\rho_{0} H}\left(t_{2}\right)+g\left(e^{-\epsilon\left|t_{1}\right|}-e^{-\epsilon\left|t_{2}\right|}\right)\left[\rho_{0 H}\left(t_{2}\right), H_{1}\right]
\end{aligned}
$$

and $g_{\epsilon} \equiv g e^{-\epsilon\left|t_{2}\right|}\left(e^{-\epsilon\left|t_{1}\right|}-e^{-\epsilon\left|t_{2}\right|}\right)$. From eq. (18), the first term is given by

$$
-\mathrm{i} \epsilon \int_{-\infty}^{0} \mathrm{~d} t_{2} e^{-\epsilon\left|t_{2}\right|} J_{\rho_{0} H}\left(t_{2}\right)=-\mathrm{i} \epsilon\left(\bar{\rho}_{\epsilon}-\rho_{0}\right) .
$$

Due to $g_{\epsilon}$, the domain of the integration of the second term in eq. (31) vanishes in the limit $\epsilon \rightarrow 0$; hence, we can omit the second term. Thus, the commutation relation in the limit $\epsilon \rightarrow 0$ becomes

$$
\lim _{\epsilon \rightarrow 0}\left[\bar{\rho}_{\epsilon}, H_{\epsilon 1}\right]=\lim _{\epsilon \rightarrow 0}-\mathrm{i} \epsilon\left(\bar{\rho}_{\epsilon}-\rho_{0}\right)
$$


On the basis of the commutation relation, the second term of eq. 30 is shown to be proportional to $\epsilon$, expressed as

$$
\begin{aligned}
\lim _{\epsilon \rightarrow 0} \operatorname{Tr}\left\{J_{\bar{\rho}_{\epsilon} H}\left(t_{1}\right) J_{c H}(t)\right\} & =\lim _{\epsilon \rightarrow 0} \operatorname{Tr}\left\{\mathrm{i}\left[\bar{\rho}_{\epsilon}, H_{\epsilon 1}\right] J_{c H}^{\prime}\left(t_{1}, t\right)\right\} \\
& =\lim _{\epsilon \rightarrow 0} \epsilon \cdot \operatorname{Tr}\left\{\left(\bar{\rho}_{\epsilon}-\rho_{0}\right) J_{c H}^{\prime}\left(t_{1}, t\right)\right\} \\
& =0,
\end{aligned}
$$

where $J_{c H}^{\prime}\left(t_{1}, t\right) \equiv U_{\epsilon}\left(t_{1}, 0\right) J_{c H}(t) U_{\epsilon}\left(0, t_{1}\right)$. The expectation values of any operator for $\bar{\rho}_{\epsilon}$ and $\rho_{0}$ are assumed to exist in the limit $\epsilon \rightarrow 0$ in Keldysh formalism. Therefore, $\epsilon \cdot \operatorname{Tr}\{\cdots\}$ vanishes in the limit $\epsilon \rightarrow 0$. In other words, $\bar{\rho}_{\epsilon}$ and $H_{\epsilon 1}$ inside the trace commute with each other in the limit $\epsilon \rightarrow 0$. We have shown that hence, the second term of eq. (30) vanishes.

Thus, we conclude that the expectation value of the current becomes independent of time in the limit $\epsilon \rightarrow 0$.

$$
\lim _{\epsilon \rightarrow 0}\left\langle J_{c H}(t)\right\rangle=\lim _{\epsilon \rightarrow 0}\left\langle J_{c}\right\rangle .
$$

Following the same type of analysis, the time-dependent correlation function of charge current is obtained as

$$
\lim _{\epsilon \rightarrow 0}\left\langle J_{c H}(t) J_{c H}\left(t^{\prime}\right)\right\rangle=\lim _{\epsilon \rightarrow 0}\left\langle J_{c H}\left(t-t^{\prime}\right) J_{c H}(0)\right\rangle .
$$

The above two equations prove that the steady state is actually reached by the adiabatic switching-on of the perturbation term in Keldysh formalism.

\section{NONEQUILIBRIUM KUBO FORMULA}

The charge current and differential conductance are respectively defined by

$$
\begin{gathered}
J(V) \equiv \lim _{g \rightarrow 1, \epsilon \rightarrow 0}\left\langle J_{c H}(t)\right\rangle=\lim _{g \rightarrow 1, \epsilon \rightarrow 0}\left\langle J_{c}\right\rangle, \\
G(V) \equiv \frac{\partial J(V)}{\partial V} .
\end{gathered}
$$

In the last equality of eq. (38), the steady-state feature of eq. (36) is used.

The differential conductance is obtained by differentiating the MacLennan-Zubarev form of the density matrix $\bar{\rho}_{\epsilon}$, eq.(20), with respect to $V$.

$$
\begin{aligned}
G(V)=\lim _{g \rightarrow 1, \epsilon \rightarrow 0} & \beta \operatorname{Tr}\left\{J _ { c } \left(e / 2\left(N_{L}-N_{R}\right)\right.\right. \\
& \left.\left.+\int_{-\infty}^{0} \mathrm{~d} t e^{-\epsilon|t|} J_{c H}(t)+\frac{\partial \Omega_{0}}{\partial V}\right) \bar{\rho}_{\epsilon}\right\} .
\end{aligned}
$$

By substituting the identity $S_{\epsilon}(0,-\infty) S_{\epsilon}(-\infty, 0) \equiv 1$, $\partial \Omega_{0} / \partial V$ is calculated as

$$
\begin{aligned}
\frac{\partial \Omega_{0}}{\partial V} & =-\operatorname{Tr}\left\{e / 2\left(N_{L}-N_{R}\right) \rho_{0}\right\} \\
& =-\operatorname{Tr}\left\{S_{\epsilon}(0,-\infty) e / 2\left(N_{L}-N_{R}\right) S_{\epsilon}(-\infty, 0) \rho_{0}\right\} \\
& =-e / 2\left\langle N_{L}-N_{R}\right\rangle-\int_{-\infty}^{0} \mathrm{~d} t e^{-\epsilon|t|}\left\langle J_{c H}(t)\right\rangle,
\end{aligned}
$$

where in the last step eq.(18) is used. Thus, we obtain the expression

$$
\begin{aligned}
G(V)=\lim _{g \rightarrow 1, \epsilon \rightarrow 0} \beta\left(\left\langle\delta J_{c} e / 2\left(\delta N_{L}-\delta N_{R}\right)\right\rangle\right. \\
\left.+\int_{-\infty}^{0} \mathrm{~d} t e^{-\epsilon|t|}\left\langle\delta J_{c H}(0) \delta J_{c H}(t)\right\rangle\right),
\end{aligned}
$$

where $\delta A \equiv A-\langle A\rangle$ and $\langle A \delta B\rangle=\langle\delta A \delta B\rangle$ are used.

Now, we rewrite the differential conductance into another expression. In preparation we derive several commutation relations. On the basis of $\left[H_{0}, e / 2\left(N_{L}-N_{R}\right)\right]=$ 0 ,

$$
S_{\epsilon}(0,-\infty)\left[H_{0}, e / 2\left(N_{L}-N_{R}\right)\right] S_{\epsilon}(-\infty, 0)=0
$$

is obtained, which leads to

$$
\begin{aligned}
& {\left[H_{0}+\int_{-\infty}^{0} \mathrm{~d} t e^{-\epsilon|t|} \mathrm{d} t J_{e H}(t),\right.} \\
& \left.\quad e / 2\left(N_{L}-N_{R}\right)+\int_{-\infty}^{0} \mathrm{~d} t e^{-\epsilon|t|} \mathrm{d} t J_{c H}(t)\right]=0
\end{aligned}
$$

From this equation, one can derive

$$
\begin{aligned}
& {\left[\bar{\rho}_{\epsilon}, H_{0}+\int_{-\infty}^{0} \mathrm{~d} t e^{-\epsilon|t|} \mathrm{d} t J_{e H}(t)\right]=0,} \\
& {\left[\bar{\rho}_{\epsilon}, e / 2\left(N_{L}-N_{R}\right)+\int_{-\infty}^{0} \mathrm{~d} t e^{-\epsilon|t|} \mathrm{d} t J_{c H}(t)\right]=0 .}
\end{aligned}
$$

Using the second commutation relation of eq.450 for the correlation functions in eq.(40), we can exchange current and the invariant part of $e / 2\left(N_{L}-N_{R}\right)$, which leads to

$$
\begin{aligned}
G(V)=\lim _{g \rightarrow 1, \epsilon \rightarrow 0} \beta\left(\left\langle e / 2\left(\delta N_{L}-\delta N_{R}\right) \delta J_{c}\right\rangle\right. \\
\left.+\int_{-\infty}^{0} \mathrm{~d} t e^{-\epsilon|t|}\left\langle\delta J_{c H}(t) \delta J_{c H}(0)\right\rangle\right) .
\end{aligned}
$$

Moreover in eqs. (42) and (46) the time integrals can be rewritten at the positive semi-infinite interval with the steady-state property of eq.(36). Thus, four distinct expressions of $G(V)$ are obtained. Their sum, divided by four, gives the symmetrized expression of differential conductance,

$$
\begin{aligned}
G(V) & =\lim _{g \rightarrow 1, \epsilon \rightarrow 0} \frac{\beta}{4}\left\langle\left\{\delta J_{c}, e\left(\delta N_{L}-\delta N_{R}\right)\right\}\right\rangle \\
& +\lim _{g \rightarrow 1, \epsilon \rightarrow 0} \frac{\beta}{4} \int_{-\infty}^{\infty} \mathrm{d} t e^{-\epsilon|t|}\left\langle\left\{\delta J_{c H}(t), \delta J_{c H}(0)\right\}\right\rangle
\end{aligned}
$$


where $\{A, B\}=A B+B A$ represents the anticommutation relation.

We find that differential conductance is determined by two contributions: the current-current correlation function and an unusual correlation function between current and the difference of the two particle numbers of reservoirs. This result directly reflects the fact that the conjugate of the bias $V$ is the invariant part of $e / 2\left(N_{L}-N_{R}\right)$ in MacLennan-Zubarev form of the density matrix. Equation (47) is the main result of this study and may be considered as the generalization of the Kubo formula in a nonequilibrium situation for a general system of a finite conductor connected to the left and right reservoirs.

\section{PHYSICAL QUANTITIES}

\subsection{Shot noise}

We introduce noise power as using

$$
S(V) \equiv \lim _{g \rightarrow 1, \epsilon \rightarrow 0} \int_{-\infty}^{\infty} \mathrm{d} t e^{-\epsilon|t|}\left\langle\left\{\delta J_{c H}(t), \delta J_{c H}(0)\right\}\right\rangle
$$

and define the current-charge correlation as

$$
S_{\mathrm{sh}}(V) \equiv-\lim _{g \rightarrow 1, \epsilon \rightarrow 0}\left\langle\left\{\delta J_{c}, e\left(\delta N_{L}-\delta N_{R}\right)\right\}\right\rangle .
$$

By using $S(V)$ and $S_{\mathrm{sh}}(V)$, the differential conductance is given by

$$
G(V)=\frac{\beta}{4} S(V)-\frac{\beta}{4} S_{\mathrm{sh}}(V) .
$$

Differential conductance and noise power are well-known observable quantities in mesoscopic systems. We propose that the $S_{\mathrm{sh}}(V)$ given by eq. (49) be defined as shot noise in general, for both interacting and non-interacting systems.

To confirm that eq. (49) is actually shot noise, we discuss the simplest example of a noninteracting Anderson model for a dot attached to the left and right reservoirs,

$$
H=\sum_{p k \sigma} c_{p k \sigma}^{\dagger} c_{p k \sigma}+\varepsilon_{d} \sum_{\sigma} n_{\sigma}+\sum_{p k \sigma} V_{p k \sigma} c_{p k \sigma}^{\dagger} d_{\sigma}+h . c .
$$

where $c_{p k \sigma}$ is the annihilation operator of the electron with the spin $\sigma$ in the left $(p=L)$ or right $(p=R)$ reservoir. $d_{\sigma}$ is the annihilation operator at the dot and $n_{\sigma}=d_{\sigma}^{\dagger} d_{\sigma}$. $V_{p k \sigma}$ is the hybridization between the dot and leads with $p=L, R$.

It is easy to calculate charge current using the Keldysh Green function

$$
J(V)=2 e \int_{-\infty}^{\infty} \frac{\mathrm{d} \omega}{2 \pi} T(\omega)\left(f_{L}-f_{R}\right),
$$

where $f_{L, R}=1 /\left(1+e^{\beta(\omega \mp e V / 2)}\right)$ are the Fermi distribution functions. Transmission probability is defined as

$$
T(\omega)=\frac{\Gamma_{L} \Gamma_{R}}{\Gamma_{L}+\Gamma_{R}} \frac{2 \Gamma}{\left(\omega-\varepsilon_{d}\right)^{2}+\Gamma^{2}},
$$

where $\Gamma_{L, R}=\sum_{k} 2 \pi\left|V_{L, R k \sigma}\right| \delta\left(\omega-\varepsilon_{L, R k}\right)$ and $\Gamma=$ $\left(\Gamma_{L}+\Gamma_{R}\right) / 2$. The differential conductance is obtained by differentiating $J(V)$ with respect $V$ as using

$$
G(V)=\beta e^{2} \int_{-\infty}^{\infty} \frac{\mathrm{d} \omega}{2 \pi} T(\omega)\left(f_{L}\left(1-f_{L}\right)+f_{R}\left(1-f_{R}\right)\right) .
$$

On the basis of the definition of eq. (48), the noise power $S(V)$ may be written as

$$
\begin{aligned}
S(V) & =4 e^{2} \int_{-\infty}^{\infty} \frac{\mathrm{d} \omega}{2 \pi} T(\omega)\left(f_{L}\left(1-f_{L}\right)+f_{R}\left(1-f_{R}\right)\right) \\
& +4 e^{2} \int_{-\infty}^{\infty} \frac{\mathrm{d} \omega}{2 \pi} T(\omega)(1-T(\omega))\left(f_{L}-f_{R}\right)^{2} .
\end{aligned}
$$

In these way, we have confirmed that noise power is given by the sum of the equilibrium noise, the first line of eq.(55), and shot noise. This result is well known in noninteracting systems 18,19 .

On the other hand, one can show that $S_{\mathrm{sh}}(V)$ is given by

$$
S_{\mathrm{sh}}(V)=4 e^{2} \int_{-\infty}^{\infty} \frac{\mathrm{d} \omega}{2 \pi} T(\omega)(1-T(\omega))\left(f_{L}-f_{R}\right)^{2}
$$

for a noninteracting system. This is nothing but the second term of eq.(55).

Therefore for the noninteracting system discussed here, we confirmed that $S_{\mathrm{sh}}(V)$ expresses shot noise and that eq. (50) actually holds.

\subsection{Generalization of Nyquist-Johnson relation}

In eq.(50), we have found that conductance is given by the difference between noise power and shot noise. We would like to stress again that this general expression is also valid in interacting systems, and not limited to the noninteracting systems discussed in the literature so far. Therefore, we propose eq.(50) as a nonequilibrium identity between physical quantities, which is a generalization of the Nyquist-Johnson relation into many-body systems in a nonequilibrium case. Below, we show that eq.(50) is actually a generalization of the Nyquist-Johnson relation.

Let us first discuss the linear response regime. The density matrix at $V=0$ can be obtained using eq.(14). It is well known that the last term of eq.(14) describes the sum of all vacuum loops, effectively giving the correction of the thermodynamic potential $\Delta \Omega \equiv \Omega-\Omega_{0} \frac{1}{*}$; hence, the equilibrium ensemble becomes

$$
\left.\lim _{g \rightarrow 1, \epsilon \rightarrow 0} \bar{\rho}_{\epsilon}\right|_{V=0}=e^{-\beta(H-\Omega)} .
$$


In any equilibrium ensemble, we can calculate the trace with the eigenfunctions of $H|n\rangle=E_{n}|n\rangle$ and $J_{c}=$ i $\left[e\left(N_{L}-N_{R}\right), H\right]$ so that

$$
S_{\mathrm{sh}}(0)=-\mathrm{i} e^{2} \sum_{n=0}^{\infty}\left\langle n\left|\left[\left(\delta N_{L}-\delta N_{R}\right)^{2}, E_{n}\right]\right| n\right\rangle=0,
$$

is derived in general. $S_{\mathrm{sh}}(0)$ vanishes in the linear response regime. Thus, we obtain

$$
4 k_{\mathrm{B}} T G(0)=S(0)
$$

This corresponds to a Nyquist-Johnson relation in the linear response regime even for systems with electron correlation 18,19 .

This relation can also be derived using the fluctuation dissipation theorem. Thus the nonequilibrium identity eq.(50) shows that a naive generalization of $4 k_{\mathrm{B}} T G(V)=$ $S(V)$ in the nonequilibrium case is not possible and there is the additional contribution $S_{\mathrm{sh}}(V)$, which plays an essential role in a nonequilibrium situation.

Finally, let us discuss how to obtain shot noise experimentally. As we have discussed in the preceding subsection for a noninteracting system, noise power may be divided into equilibrium noise and shot noise. Experimentally, we measure only the noise power. In actual cases, it has been discussed that noise power for $e V>k_{\mathrm{B}} T_{K}$ includes shot noise significantly. On the other hand, the nonequilibrium identity suggests that shot noise as the difference between noise power and differential conductance expressed as

$$
S_{s h}(V)=S(V)-4 k_{\mathrm{B}} T G(V),
$$

both of which are measurable quantities. Thus, we may use this identity as the definition of shot noise for both interacting and noninteracting systems.

\section{CONCLUSION}

We have clarified that the density matrix obtained by the Keldysh formalism of a finite conductor in series with reservoirs is an example of MacLennan-Zubarev ensembles. On the basis of this fact, we pointed out that the adiabatic switching-on of the perturbative term of Keldysh formalism corresponds to taking the invariant part with the causality condition introduced by Zubarev. From this fact, we conclude that Keldysh formalism may describe an irreversible process and the steady-state feature of the time-correlation functions For proof of this steady-state feature, it is essential to understand the fact that the density matrix and total Hamiltonian commute in the expectation values.

Using the MacLennan-Zubarev form, we have derived the general expression of differential conductance, which is determined by the current-current correlation function and the correlation function between current and the difference of the two particle numbers of reservoirs. We call it the nonequilibrium Kubo formula of a finite conductor attached to reservoirs. It is well known that noise power is given by the current-current correlation function. We have proposed that the correlation function between current and the difference of the two particle numbers of reservoirs gives the shot noise of the system, in general. This fact is confirmed by explicit calculations using the noninteracting Anderson model. Therefore, we propose that the nonequilibrium identity between differential conductance, noise power and shot noise is a generalization of the Nyquist-Johnson relation in general.

\section{Acknowledgments}

The author would like to thank K. Ueda, T. Kato and K. Saito for valuable discussions. This research was partially supported by the Ministry of Education, Science, Sports and Culture, Grant-in-Aid for Young Scientists (B), 2005, 17740187.
1 L. V. Keldysh: Sov. Phys. JETP. 20 (1965) 1018.

2 D. C. Langreth: in Linear and Nonlinear Transport in Solids, eds. J. T. Devreese and V. E. Van Doren (Plenum Press, New York, 1976) Vol. 17 of NATO ASI, Series B: Physics.

3 J. Rammer: Rev. Mod. Phys. 58 (1986) 323.

4 A. Kamenev: in Strongly Correlated Fermions and Bosons in Low-Dimensional Disordered Systems, eds. I. V. Lerner, B. L. Althsular, V. I. Fal'ko, T. Giamarchi (Kluwer Academic Publishers, Dordrecht, 2002) Nato Science Series; Sub-series II, Mathematics, Physics and Chemistry, Vol 72.

5 A. Kamenev: in Lectures notes for 2004 Les Houches Sum- mer School on "Nanoscopic Quantum Physics".

6 S. Hershfield: Phys. Rev. Lett. 70 (1993) 2134.

7 A. Schiller and S. Hershfield: Phys. Rev. B 51 (1995) 12896; A. Schiller and S. Hershfield: Phys. Rev. Lett. 77 (1996) 1821; A. Schiller and S. Hershfield: Phys. Rev. B 58 (1998) 14978.

8 S. Tasaki: Chaos, Solitons and Fractals 12 (2001) 2657; in Statistical Physics, ed. M. Tokuyama and H. E. Stanley (AIP Press, New York, 2000) p. 356; in Quantum Information III, ed. T. Hida and K. Saito (World Scientific, Singapore, 2001) p. 157.

9 S. Tasaki and T. Matsui: in Fundamental Aspects of Quantum Physics, eds. L. Accardi and S. Tasaki (World Scien- 
tific, 2003) p. 100

10 J. Takahashi and S. Tasaki: J. Phy. Soc. Jap. 75 (2006) 94712.

11 C. Caroli, R. Combescot, P. Nozières, and D. S-James: J. Phys. C: Solid St. Phys. 4 (1971) 916.

12 S. Hershfield, J. H. Davies, and J. W. Wilkins: Phys. Rev. Lett. 67 (1991) 3720; Phys. Rev. B. 46 (1992) 7046.

13 Y. Meir, N. S. Wingreen, and P. A. Lee: Phys. Rev. Lett. 70 (1993) 2601; N. S. Wingreen and Y. Meir: Phys. Rev. B. 49 (1994) 11040.

14 P. Nozières: Theory of Interacting Fermi Systems (Benjamin New York, 1964).

15 See, for example, A. L Fetter and J. D. Walecka: Quantum
Theory of Many Particles (McGraw-Hill, New York, 1971).

16 D. N. Zubarev: Nonequilibrium Statistical Thermodynamics (Consultants, New York, 1974).

17 J. A. MacLennan, Jr.: Adv. Chem. Phys. 5 (1963) 261.

18 M. J. M. de Jong and C. W. J. Beenakker: in Mesoscopic Electron Transport, eds. Lydia L. Sohn, Leo P. Kouwenhoven, and Gerd Schon (Kluwer Academic Publishers, Dordrecht, 1997) Vol. 345 of NATO ASI, Sereies E: Applied Sciences, p. 225.

19 See, for example, Ya. M. Blanter and M. Büttiker: Physics Reports 336 (2000) p. 1. 